\title{
L'avenir de la Suisse urbaine: changement technologique, métropoles informationnelles et développement territorial
}

\begin{abstract}
Résumé
L'évolution du système urbain helvétique est en partie conditionnée par sa position au sein du réseau de polarités qu structurent le continent. Dans cette espace multipolaire, la globalisation de l'économie, l'innovation technologique, l'avènement d'une nouvelle culture individualiste, la crise de légitimité de l'Etat, la formation de régions métropolitaines, la dégradation de l'environnement dessinent déjà des figures possibles de l'avenir. La Suisse n'est pas à l'abri de ces turbulences. L'incertitude qui pèse sur les résultats de la dynamique urbaine incite à la mise en œuvre de politiques territoriales globales, actives et préventives.
\end{abstract}

C'est généralement dans les périodes de grands changements que les pays apprennent le mieux à reconnaître leur territoire. Dans le contexte problématique créé par la mondialisation des échanges, l'intégration européenne, le changement technologique accéléré et la montée des métiers de type informationnel, les régions, les villes et les agglomérations helvétiques seront plus que jamais exposées à des tensions et à des transformations. Des logiques économiques, politiques et culturelles mondiales, européennes, transfrontalières, nationales, régionales et locales sont à l'œuvre imprimant de nouvelles configurations économiques et sociales, déjà perceptibles, dans les paysages urbains fortement tertiairisés. Les grandes villes se délayent dans des régions urbaines de plus en plus dilatées. Mais ces transformations économiques et morphologiques tendent à capitaliser les avantages comparatifs des grandes régions urbaines. Dans l'analyse territoriale, le nouveau apparaît fortement structuré par l'ancien. L'avenir territorial de la Suisse s'inscrit déjà dans la diversité des formes urbaines et des hiérarchies spatiales actuelles.

Le changement dans l'invariance: telle est notre hypothèse de travail principale. C'est le renouvellement des dispositifs de la centralité urbaine qui structure toujours les choix de localisation des acteurs économiques, les rythmes de fréquentation des lieux et l'ensemble du système de relations des habitants à leur territoire. Le caractère de plus en plus informationnel de la plupart des activités tertiaires, la diversification et la formation du capital humain sont, pour de nombreuses villes, des sources d'avantages comparatifs. L'efficacité économique des entreprises repose de plus en plus sur les dispositifs urbains de l'intelligence économique. Dans cette perspective, le niveau et la diversite des qualifications du travail sont des facteurs stratégiques du développement à long terme.
Les villes se métamorphosent, mais leurs positions relatives dans la hiérarchie urbaine demeurent inchangées. Il reste que le temps peut toujours nous révéler un ordre territorial interdit à nos représentations partielles. Aussi conviendrait-il d'élaborer des stratégies de développement territorial qui soient assez fermes pour maitriser les effets négatifs des changements prévisibles, mais assez souples pour s'adapter aux conditions locales et à l'imprévu résultant du jeu de tendances contradictoires et partiellement inconnues. Le changement économique et technologique est toujours ambivalent. La permanence des déséquilibres territoriaux, la crise écologique, la crise de l'espace urbain en tant que cadre de vie et support de la solidarité sociale et spatiale sont là pour nous rappeler à une vigilance permanente. Sans doute l'évolution des structures territoriales dépendra-t-elle aussi des projets collectifs que nous saurons élaborer et réaliser. A la veille du troisième millénaire, la nature et les incertitudes des défis urbains, régionaux et européens plaident pour une réhabilitation et un renouveau des objectifs et des instruments de la régulation territoriale.

\section{Entre passé et devenir: changement dans l'invariance et rôle clé des qualifications du travail}

L'avant-crise nous a légué le développement inégal, l'exode rural, l'explosion suburbaine, la dégradation du milieu, la disparition des projets de société porteurs d'avenirs et d'espoirs. Quant à la crise, elle nous a révélé l'avènement de la nouvelle culture individualiste, l'émergence des nouvelles technologies, la globalisation de l'économie, l'intensification de la division du travail, le chômage et l'exclusion sociale, mais aussi la carence des pouvoirs publics quant à la définition d'objectifs et de politiques à long terme. Désindustrialisation relative ou absolue, tertiairisation sélective, concentration des entreprises sont des processus qui qualifient les changements structurels intervenus dans le système productif helvétique au cours des quinze dernières années (CUNHA, 1993, CUNHA et RACINE, 1994).

Antonio Cunha, Chargé de cours, Institut de Recherche sur l'Environnement Construit, Département d'Architecture Ecole Polytechnique Fédérale de Lausanne. 


\section{Désurbanisation ou changement de régime d'urbanisation?}

On se souvient de la polémique engendrée par les représentations "prophétiques» proposées en regard de la saisie, formelle et statistique, de l'accélération de l'étalement des aires urbaines en Occident, représentations évoquant tour à tour un processus de désurbanisation et de contre-urbanisation pour décrire la déprise démographique des grandes villes et la croissance corrélative de la population des périphéries proches ou lointaines (BERRY, 1976; KLAASSEN et SCIMENI, 1981). Force est de reconnaître, en Suisse comme ailleurs, qu'on en avait conclu un peu vite que la dynamique de l'urbanisation, en marche depuis la révolution industrielle, était arrivée à son terme.

En dépit du ralentissement relatif de la croissance des zones urbaines par rapport aux communes non urbaines (hors-réseau), le processus de suburbanisation ne s'est pas arrêté. C'est ce que montre le graphique de la fig.l. Il permet de déceler grossièrement une période d'urbanisation qui se prolonge jusqu'aux années quarante et une période de suburbanisation relative qui s'étend jusqu'à la fin des années septante. Depuis le début des années septante, on est entré dans une phase de suburbanisation absolue, couplée à un processus de périurbanisation: c'est la combinatoire de ces deux processus qui donne l'illusion d'un processus de désurbanisation à l'échelle de l'ensemble territorial helvétique.

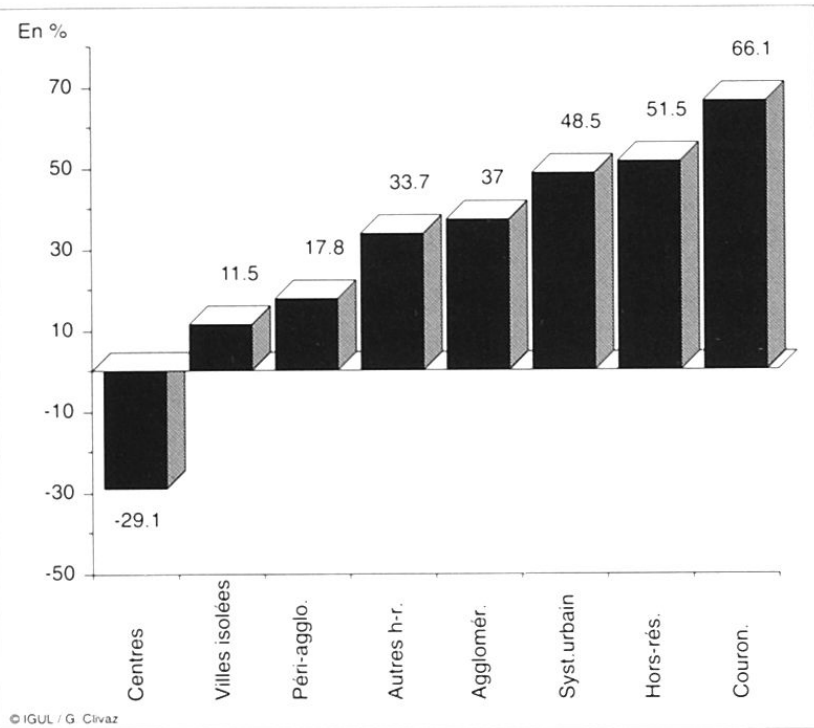

Fig.1 Evolution des poids démographiques selon la zone urbaine, 1850-1990.

L'analyse a permis de montrer que la dernière phase coïncide avec une période de forte désindustrialisation, phase aussi de reterritorialisation des centres et des périphéries par l'extraordinaire expansion quantitative et qualitative du tertiaire. Elle est caractérisée par un nouveau ralentissement du système urbain et par un changement dans le régime d'urbanisation probablement destiné à se prolonger dans les années à venir.
Ce changement de régime est à mettre en relation avec cinq facteurs au moins: la décision prise en 1964 par le gouvernement fédéral de limiter la demande globale notamment par des restrictions à l'immigration (ROSSI, 1983 ) certes, mais aussi la désindustrialisation progressive du système productif et l'investissement sélectif des centres par des activités tertiaires à haute valeur ajoutée, l'accroissement global des niveaux de revenus, l'accroissement de la mobilité et le changement des valeurs et des modes de vie des populations. Pourtant, le processus de suburbanisation engagé depuis la fin de la Seconde Guerre mondiale se poursuit, les couronnes continuant à augmenter leur poids démographique compensant ainsi largement les pertes des centres (cf. fig. 2).

\section{La formation des régions urbaines et métropolitaines: spécialisations tertiaires et qualifications du travail}

L'urbanisation se poursuit selon des modalités de concentration urbaine fondées sur un affinage progressif des centres. Des régions urbaines, des ensembles urbanisés diversifiés, formés de villes industrielles ou tertiaires de taille moyenne ou petite, coupés parfois de césures agricoles, émergent autour des grandes villes. Mais la constitution de ces nouvelles configurations urbaines n'est qu'une expression du renforcement du rôle économique et social, à l'échelle du pays, des métropoles anciennes. Une série d'analyses multidimensionnelles, conduites tant en coupe que longitudinalement, a confirmé que ces dernières sont bien installées au sommet de la hiérarchie (CUNHA, 1993): deux régions métropolitaines et trois régions urbaines en fait, qui polarisent l'essentiel des dynamismes démographiques, économiques et technologiques du territoire helvétique; leur développement résulte de la combinatoire de processus centre - périphérie et de processus de métropolisation qui continueront certainement de jouer un rôle dans les années à venir.

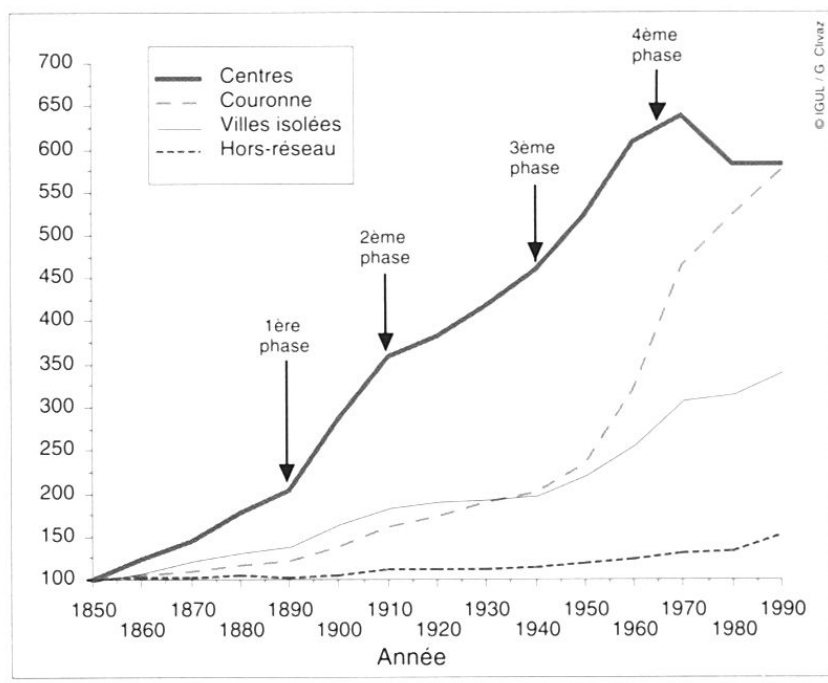

Fig. 2 Part relative dans la croissance démographique seIon la zone (en \% de l'accroissement démographique global enregistré en Suisse entre 1970 et 1990). 
La région métropolitaine aréolaire de Zurich: organisée autour de la plus grande ville suisse et des communes qui constituent sa couronne périphérique, elle se prolonge sur trois axes autoroutiers jusqu'à Baden, Winterthour et Zoug et elle regroupe une deuxième couronne de petites villes périphériques en croissance, généralement spécialisées dans l'activité industrielle (cf. aussi SCHULER, 1994).

La région métropolitaine bipolaire du Léman: c'est une région à dominante tertiaire. Elle est constituée par quatre pôles secondaires, Yverdon, Vevey-Montreux, Morges et Nyon, ces deux derniers centres étant intégrés dans les agglomérations de Lausanne et de Genève (fig. 3). Ensemble ces pôles concentrent environ $18 \%$ de la population urbaine.

La région à urbanité diffuse du Tessin: à dominante tertiaire elle est structurée par quatre pôles, chacun d'entre eux spécialisé dans une activité tertiaire spécifique. L'agglomération de Lugano, spécialisée dans les services financiers et d'une manière plus générale dans les services aux entreprises, occupe une place originale au sein de la région en tant que point d'articulation entre les espaces italiens et alémaniques.

Les agglomérations et régions urbaines unipolaires de Berne et de Bâle: ces deux grands pôles complètent le niveau supérieur de l'armature urbaine.
Aux niveaux inférieurs de la hiérarchie urbaine ont retrouve l'ensemble formé par les agglomérations de taille moyenne et petite et l'ensemble des petites villes isolées: Les agglomérations moyennes et petites: localisées aux limites ou à l'extérieur des trois régions urbaines mentionnées, elles constituent les trois niveaux inférieurs de la hiérarchie urbaine. Ensemble elles constituent la véritable colonne vertébrale du territoire helvétique, structurant le ruban urbain du Moyen-Pays. D'autres sont des centres de taille relativement modeste (25 000 à 75000 ) mais qui jouent un important rôle à l'échelle régionale (Coire, Liestal, Olten, Arbon-Rorschach, etc.).

Les villes isolées et les petites unités interstitielles: leurs populations varient entre 5000 et 25000 habitants. Elles animent les différents espaces périphériques. En fait, trois types de "situations périphériques" peuvent être décelées: petits centres de la périphérie des régions urbaines; centres des régions industrielles périphériques; centres des périphéries agro-touristiques.

Les cinq plus grandes agglomérations qui organisent les régions urbaines et métropolitaines ont drainé à elles seules plus de la moitié du solde des emplois créés entre 1975 et 1991. Elles entretiennent entre elles des liens privilégiés d'échange que l'on peut lire à travers les choix des localisations des grandes entreprises à établissement unique ou à multi-établissements (ROSSI et PINI, 1994).

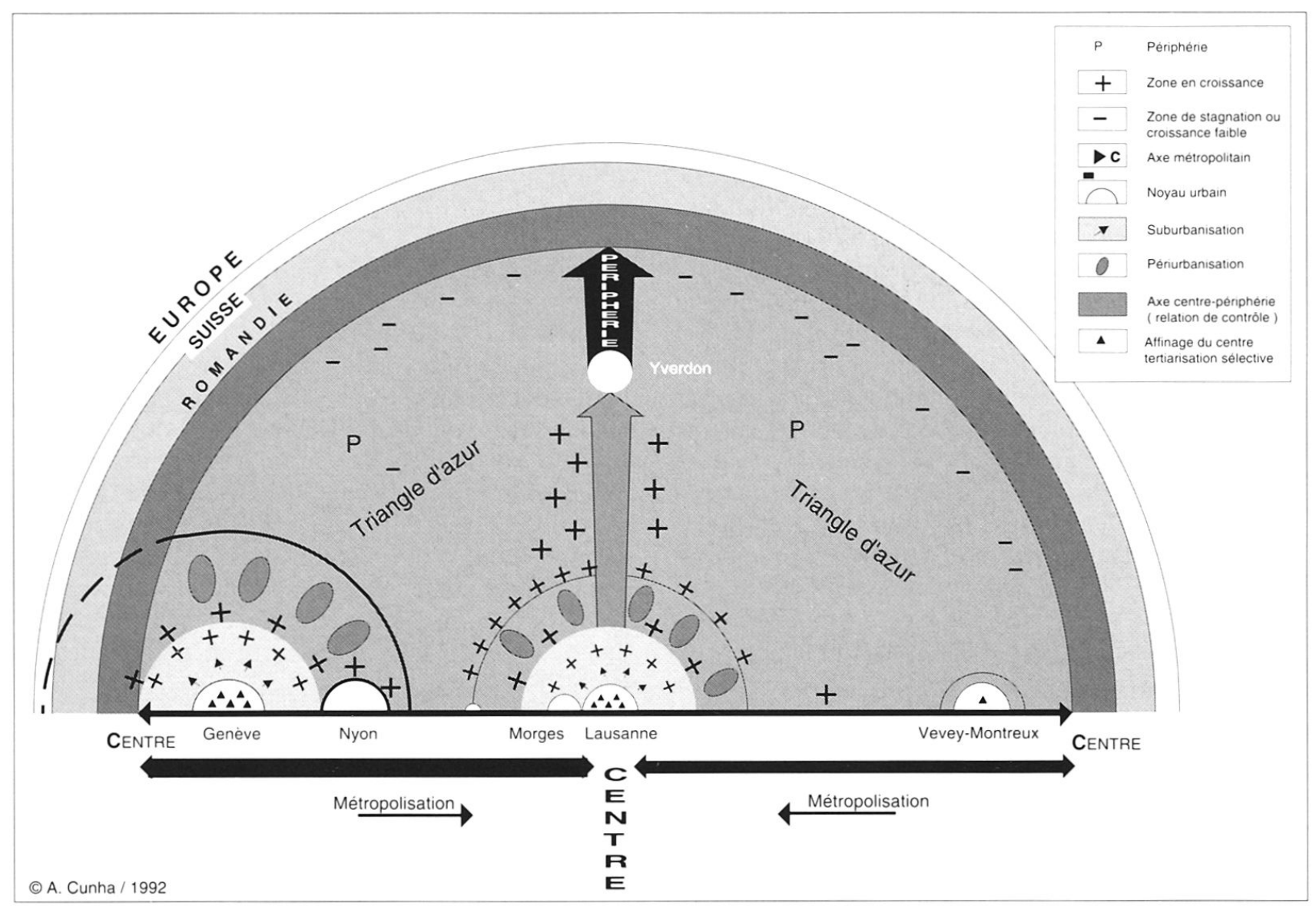

Fig.3 Schéma structurel de la région métropolitaine du Léman. 
Relativement massive, la polarisation de l'emploi reste différentielle (cf. fig. 4). L'analyse menée à l'échelle de l'armature urbaine montre que la hiérarchie des polarisations de l'emploi coüncide étroitement avec la hiérarchie des qualifications du travail. Le contenu sans cesse plus informationnel de la plupart des activités tertiaires rend la diversification et la formation du capital humain une source vitale d'avantages comparatifs pour les grandes villes.

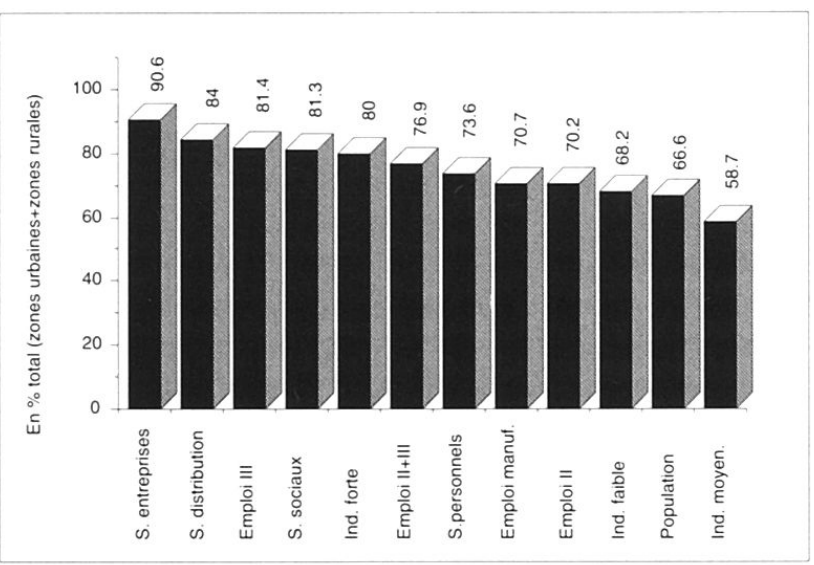

Fig. 4 Croissance de l'emploi dans les zones urbaines, 1975-1985.

La diversité des ressources humaines, leur niveau de formation, leur compatibilité linguistique, leur mobilité, leur familiarité avec les pratiques d'affaires nationales et internationales, etc. sont des atouts majeurs pour les métropoles (DANIELS, 1993). Un constat qui permet de mieux spécifier la notion de centralité urbaine, montrant bien que le pouvoir décisionnel de ces villes continue à s'étendre sur l'ensemble du territoire national malgré le ralentissement démographique de leurs villes-centre.

En Suisse, le gradient observé des niveaux de formation et de qualification selon la taille des villes et agglomérations est quasiment linéaire: seules les cinq grandes agglomérations présentent des niveaux de formation supérieurs à la moyenne du réseau. En revanche, la part de la population n'ayant suivi qu'une formation de premier degré est surreprésentée dans les communes de 5000 à 25000 habitants (41 à $44,4 \%$ de la population pour un taux moyen de $34,7 \%$ pour l'ensemble du réseau).

La structure socio-professionnelle est en étroite corrélation avec ce résultat. Dans les villes de petite taille, les ouvriers et artisans sont nettement surreprésentés. Les grandes villes, en général plus tertiairisées, accueillent les employés mais aussi les cadres, les dirigeants d'entreprise et les professions libérales: à ville plus grande, travail plus qualifié.

Bref, l'analyse révèle l'accroissement des disparités qualitatives en relation avec la tertiairisation sélective et hiérarchique des centres urbains dans les services les plus performants et à plus forte valeur ajoutée et la «décon- centration" des services banals et des activités manufacturières en direction des périphéries proches ou lointaines. Ces processus sélectifs tendent à engendrer des types d'associations d'activités tertiaires et manufacturières assez nettes qui expriment des modes de développement caractéristiques de l'émergence de la ville informationnelle.

Les centres des régions centrales paraissent offrir des surplus de localisation pour les entreprises (économies d'agglomération nettes positives) ayant une importante incidence sur la polarisation des activités les plus dynamiques. En revanche, les villes et agglomérations moyennes ne paraissent pas jouer le rôle de relais que la théorie leur accorde généralement, leur développement durant la dernière période d'expansion ayant été freiné par un profil de spécialisation économique défavorable (textil, habillement, horlogerie, etc.). Ce résultat est en grande partie incompatible avec l'hypothèse de plus en plus souvent proposée d'un «retournement de tendance». L'analyse des profils de spécialisation et des positions des zones urbaines et des régions tend plutôt à confirmer l'idée d'un changement général des structures économiques dans l'invariance des positions territoriales.

\section{La Suisse au milieu des turbulences: globalisation, modernisation technologique et crise de l'espace urbain comme support de la solidarité}

La Suisse est au cœur de la mégalopole européenne et l'évolution de son territoire est en partie conditionnée par sa position au sein du réseau de polarités, de voies de communication et d'échanges qui structurent le continent. Elle y occupe une position qui inscrit ses villes et ses régions dans un système d'interdépendances complexes. Plusieurs composantes du changement de l'environnement territorial influencent son développement.

\section{Les composantes du changement: globalisation de l'économie, division du travail, individualisme, dérégulation et métropolisation}

L'internationalisation des échanges économiques est une des composantes les plus visibles du changement des nations et des régions de l'Europe. Certes, elle n'est pas récente mais elle s'accélère et se globalise depuis quelques décennies en particulier en raison de la construction européenne et de la mise en œuvre de formes de régulation économique communes. Cette globalisation élargit et emboîte les échelles spatiales par la structuration des firmes en réseaux, en chaînes et filières de production déterminant partiellement le fonctionnement des villes et des régions.

La concurrence internationale est source de dynamisme mais elle sanctionne aussi, implacablement, tout retard dans l'innovation technologique territoriale. La compétitivité variable des activités économiques sur les marchés externes a pour résultat des taux de croissance régionaux différentiels et, parfois, un accroissement des inégalités territoriales. L'internationalisation ne concerne pas que les firmes, les modalités de régulation économique et les 
techniques. Elle concerne également les individus, de plus en plus mobiles, aussi bien dans leurs métiers que dans leurs loisirs. Les touristes et les immigrés apportent aussi une culture. Les brassages, les métissages renforcent la diversité et la communication interculturelles. Ils génèrent parfois des comportements de repli et des situations de conflit potentiel.

Deuxième axe du changement: l'intensification de la division du travail. A mesure que l'internationalisation de l'économie progresse et que le changement technologique diffuse, on constate l'accroissement de la productivité dans certaines branches et la perte d'emplois dans d'autres. La transition vers la société de services est bien au cœur de tous les processus participant à la transformation économique d'ensemble: spécialisation dans les branches industrielles progressives, intégrant prioritairement capital humain et information dans la recherche d'une valorisation continue de l'innovation; croissance du tertiaire affilié; multiplication des unités techniques spécialisées; augmentation des échanges, des circulations et des communications de toute nature; émergence de modes de produire de plus en plus flexibles liés à la désintégration verticale et à la disjonction fonctionnelle au sein des firmes. Mais de l'intensification de la division du travail et de la montée des professions qualifiées naissent aussi des exclusions. Le marché du travail peut devenir une extraordinaire fabrique d'exclus qui ne sélectionne que ceux qui possèdent les qualifications requises pour les nouvelles tâches productives.

La culture est un autre grand axe de changement. Le brassage des cultures n'est pas le seul facteur visible. Probablement, ce n'est pas le plus important. Le mouvement de fond qui caractérise la grande transformation culturelle des démocraties européennes est l'avènement d'une nouvelle culture individualiste. Les utopies ont changé d'échelle. Les modèles socio-culturels sont désormais multiples, optionnels et également légitimes. La pratique de la ville devient un jeu d'identifications multiples. L'identité culturelle devient une notion de plus en plus lièe à des choix personnels. Certes, l'individualisme peut générer l'indifférence. Mais la disqualification individualiste des grandes utopies historiques et la valorisation de "l'accomplissement de soi» apparaissent de plus en plus souvent conjuguées avec des préoccupations de responsabilité envers les autres, envers la nature et l'environnement. Autant d'éléments susceptibles d'engendrer une nouvelle culture politique.

Quatrième axe de changement: l'Etat. L'internationalisation de l'économie rend les économies nationales de plus en plus interdépendantes et limite les degrés de liberté des administrations de plus en plus contraintes par des réglementations supranationales. En même temps l'individualisme met l'Etat en crise de légitimité. Dans l'ambiance néolibérale qui règne très largement encore aujourd'hui, certains voudraient limiter l'intervention publique à des démarches ciblées et consensuelles visant surtout à accompagner les dynamiques spontanées du marché par une politique d'encadrement. Mais si certains prônent l'Etat minimal - qui ne devrait intervenir que là où des acteurs privés ne peuvent le faire -, il en est d'autres qui appellent à son renouvellement et à son redéploiement aux échelles locales et régionales. Dans cette perspective, l'intervention publique décentralisée devrait désormais pallier l'incapacité de l'initiative privée à susciter les nécessaires régulations intersystémiques. Les acteurs sociaux du développement resteront encore partagés entre la nécessité d'une dérégulation et l'exigence d'une rerégulation.

Enfin, dernière composante majeure des changements en cours: les transformations de l'espace lui-même. La division du travail et la ségrégation fonctionnelle et spatiale des processus de production industrielle intensifient les relations lointaines et accroissent le rôle des technologies de maitrise de la distance. Si, pour certains, les nouvelles technologies de communication offrent une nouvelle chance aux régions périphériques, d'autres pensent, au contraire, que la télématique ne pourra pas supplanter le face-à-face. Les moyens de communication et de télécommunication ne sont pas des substituts parfaits et la diffusion des nouvelles technologies de maîtrise de la distance ne ferait que renforcer le rôle des métropoles anciennes. L'émergence de nouvelles filières de production met de nombreuses régions incapables de suivre le cycle des innovations en congé de croissance. En revanche, les grandes villes se délayent dans des espaces périphériques de plus en plus éloignés. Partout on se demande comment compatibiliser les logiques de développement hétérocentrées, axées sur l'accessibilité aux réseaux externes de complémentarité, et les exigences d'un développement local, territorial qui tend à apparaître comme une réponse possible aux questions posées dans un monde en profonde mutation.

\section{La Suisse au milieu des turbulences: le scénario plus probable}

La Suisse ne vit pas à l'abri de ces changements, de ces turbulences et de ces ruptures. Quel que soit le résultat des négociations et décisions à venir, la Suisse ne peut éluder ni son intégration de fait à l'économie européenne, ni son emboîtement dans les réseaux européens. En dépit de la concurrence des coûts de production, l'industrie suisse assurera certainement une place importante dans la course aux nouvelles technologies. Dans le secteur secondaire, l'automatisation et la rationalisation de l'industrie tendent à se poursuivre, augmentant la productivité du secteur manufacturier mais en réduisant l'emploi de manière significative. La désindustrialisation relative de l'appareil productif est une tendance lourde qui se renforce avec les comportements de délocalisation et d'investissement des grandes entreprises à l'étranger; corrélativement, la tertiairisation et l'accroissement généralisé du niveau de qualification du travail s'affirmeront encore davantage.

Nombre de facteurs qui expliquent l'expansion régulière de l'emploi tertiaire depuis les années 60 (transformations des modes de vie, éclatement de la famille traditionnelle, féminisation du travail, vieillissement de la population, 
«externalisation» du tertiaire affilié, accroissement de l'importance des secteurs produisant de l'information, importance de la recherche-développement, etc.) continuent à jouer un rôle aujourd'hui, malgré un infléchissement remarqué de la croissance de l'économie des services.

Les tendances et les mutations probables dans les domaines économique, technique, culturel et politique peuvent avoir des effets spatiaux contradictoires: certains changements peuvent soit favoriser la dispersion des activités et des hommes, soit accroître leur concentration au sein de nouvelles entités territoriales; à l'échelle locale, une concentration des emplois est compatible avec une dispersion de la population; la diminution de la densité des grandes villes-centres peut se poursuivre parallèlement à une concentration de la population à l'échelle nationale au sein de nouvelles régions urbaines; certaines évolutions dans les technologies de communication peuvent aussi bien accroître la fréquence des déplacements de personnes que la réduire, etc. Si certaines villes sont, aujourd'hui, menacées de déclin économique et démographique, d'autres pourront, au contraire, continuer à tirer pleinement profit des mutations en cours, assurant ainsi les positions conquises dans le passé. Quel semble être alors le scénario le plus probable?

En dépit d'une diffusion sans précédent des nouvelles technologies de maîtrise de la distance, l'intégration et la spécialisation croissantes de l'économie contemporaine aux différentes échelles d'analyse tendent à fixer progressivement des activités tertiaires de commandement et de gestion économique dans un petit nombre de grands centres pouvant offrir un large éventail de professions à haute qualification. Le scénario de la globalisation, de la spécialisation économique, de la société informationnelle, de la compétition technologique est aussi celui de la métropolisation croissante du territoire.

Le territoire en gestation s'apparente à un système de plus en plus ramifié et interdépendant de zones urbaines et métropolitaines avec des centres spécialisés, des centres-relais, des lieux clefs et des lieux foyers, mais aussi de fines capillarités oủ continue à fonctionner l'interactivité centre - périphérie. La métropolisation est inscrite dans les tendances actuelles que nous avons présenté précédemment; elle doterait la Suisse d'une, voire deux grandes métropoles européennes et elle renforcerait le rôle des régions zurichoise et lémanique dans un contexte d'internationalisation croissante.

La recomposition du territoire autour des grands centres urbains et de leurs réseaux ne peut être qu'amplifiée par le processus d'intégration au système de communications européen. Pour répondre à une demande accrue de mobilité, la Communauté envisage le développement rapide de liaisons de grande qualité entre les principaux centres urbains, y compris naturellement des liaisons ferroviaires à grande vitesse. Comme le souligne G. WACKERMANN (1991), ces cheminements susciteront de nouvelles solidarités spatiales, multiples, de moins en moins juxtaposées et de plus en plus emboîtées. Les flux de biens et de personnes, actionnés à travers des réseaux par des pôles métropolitains à vocation européenne et mondiale, conduiront à un inévitable élargissement des échelles.

Ces développements sont vitaux pour l'avenir des régions et des villes suisses. Les facteurs d'hétérogénéité spatiale et donc les clefs du développement de demain ne seront pas les informations téléaccessibles, mais au contraire celles auxquelles les nouvelles technologies de maîtrise de la distance ne permettent pas d'accéder directement. La connectivité des réseaux matériels de communication continuera à être décisive pour parvenir aux informations non médiatisables. Cette hypothèse de travail spécifie les objets et les qualités qui donneront un avantage comparatif aux villes de demain: les dispositifs les plus spécialisés et les plus rares de la centralité économique, culturelle et politique et les qualifications du travail qui leur sont associées. La diffusion généralisée des outils de télécommunication pourrait donner, paradoxalement, un avantage comparatif considérable aux grandes agglomérations, à condition qu'elles parviennent à renouveler les fonctions économiques qualifiées et qualifiantes.

Mondialisation des échanges et des entreprises, transformation du système productif, rôle stratégique de la connaissance au sein de schémas de production flexibles, évolution des technologies de maîtrise de la distance, tendance à la concentration du capital technologique, scientifique et tertiaire avancé, logique concentrationnaire des téléports, aéroports et transports à grande vitesse, mais aussi changements de la structure des familles et monoparentalisme croissant, vieillissement démographique, réduction des dépenses sociales de l'Etat. Telles sont les principales tendances à l'œuvre.

Cette évolution s'inscrit bien dans une logique fonctionnelle de communication et non dans une logique de solidarité sociale et spatiale. C'est une logique productiviste, informationnelle, transactionnelle, qui s'inscrit dans un scénario fondé sur des espaces d'échange branchés sur les grands réseaux et autres autoroutes de la communication-information. Scénario moderniste de la métropolisation à outrance, traduisant un type de développement qui peut engendrer des effets spatiaux et sociaux tout aussi visibles, qui s'inscrivent aussi et déjà dans les réalités quotidiennes. Quels problèmes pose-t-il?

Du point de vue de l'évolution de la structure urbaine, l'affaiblissement des villes moyennes, affaiblissement de la base économique et démographique des villes et agglomérations spécialisées dans les branches exposées à la concurrence internationale apparaît comme la conséquence la plus probable. Mais les risques d'une dissociation progressive des espaces périphériques sont aussi très présents dans un scénario qui tendrait à coupler l'internationalisation économique de fait avec une croissante automarginalisation politique par rapport aux centres de décision européens.

Dans cette variante du scénario métropolitain les grandes villes mais aussi les régions transfrontalières (cf. fig. 5) chercheraient à se profiler comme partenaires 
d'un centre extérieur comme Lyon, Turin, Milan, Munich ou Stuttgart; Bâle est déjà intégrée à la région du HautRhin. Le canton de St-Gall et une partie de la Suisse orientale verront leur croissance influencée favorablement par des impulsions données par la région du Lac de Constance. Le canton du Tessin a une politique extérieure tendant à instituer une coopération régionale dans l'Arc alpin et en Europe. La collaboration avec la région à forte croissance qu'est l'Italie du Nord devrait porter des fruits. Les relations avec la région française Rhône-Alpes seront primordiales pour Genève et pour l'ensemble de la région métropolitaine du Léman qui pourrait éventuellement jouer un rôle charnière pour le reste de la Suisse romande et du pays.

Cependant, les enjeux d'une métropolisation et d'une intégration européenne non maîtrisées ne sont pas uniquement spatiaux : la déconnection du tissu social, l'absence de véritable politique d'intégration des populations étrangères, l'émergence de la pauvreté d'exclusion au sein des différentes minorités, ethniques et sociales (familles monoparentales, vieux, chômeurs), l'augmentation de l'individualisme et la perte du sens, du relationnel, et des identités communautaires, le maintien ou la progression des bureaucraties administratives dans leurs logiques purement sectorielles, et l'impossibilité d'élaborer un projet global pèsent dangereusement sur le scénario productiviste. De nouvelles polarisations, bien connues chez nos voisins, s'inscrivent dans un phénomène plus général de partition qui renvoie lui-même à l'opposition entre exclusion et participation. La nouvelle société urbaine est de plus en plus considérée comme une "fabrique d'exclus» (TOURAINE, 1991).

Le chômage de longue durée fait désormais partie du paysage économique et social helvétique. L'invention de nouveaux dispositifs d'insertion économique et sociale devient une priorité absolue si nous voulons faire face à l'exclusion. Même dans les pays occidentaux les plus avancés et les plus riches, le constat s'impose: la société à deux vitesses devient la composante dominante de la structuration sociale et spatiale. Il ne s'agit évidemment plus seulement de découvrir les inégalités. Elles existent en Suisse aussi. La question est de savoir si nous sommes dans une société qui va de plus en plus spatialiser ces inégalités et qui va les rendre dans une certaine mesure irréversibles ou au contraire dans une société qui va pouvoir les traiter, en se fondant sur les valeurs de dignité et d'enracinement. On peut se demander si notre société porte en elle-même la capacité de réduire ces écarts et surtout si elle n'y pousse pas par ses propres divisions du travail, par ses propres status modifiés du travail, par son propre systeme de valeurs. La vraie question est sans doute de savoir ce que devient alors notre idéal fédéraliste et dé-

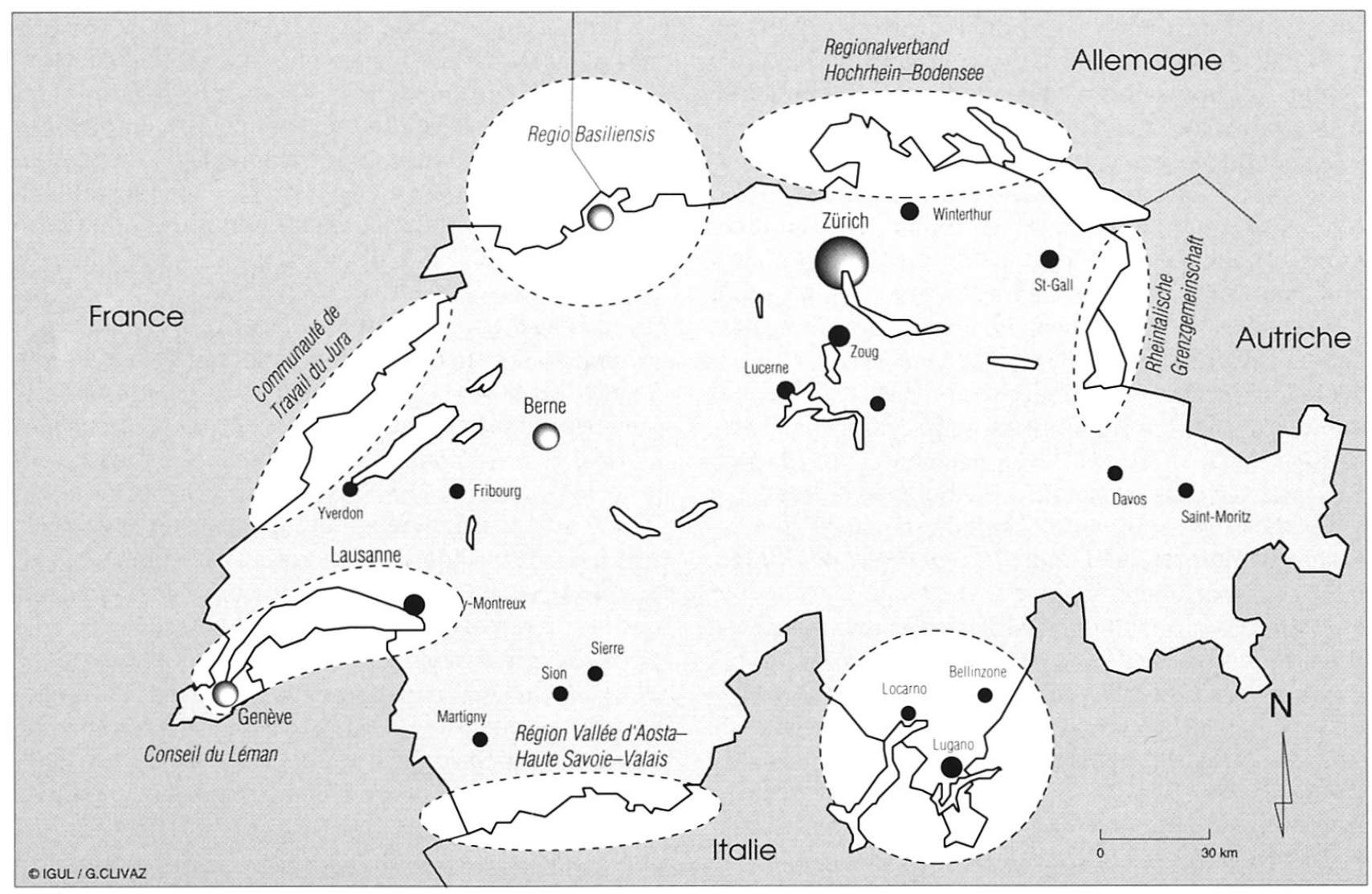

Fig. 5 Les régions transfrontalières. 
mocratique, qui suppose une "égale dignité de tous" (RACINE, 1992). Que peut-il signifier au double plan des hommes et des territoires? C'est en tout cas l'une des dimensions du problème de l'avenir des villes auquel il nous faut continuer de réfléchir.

Ne serait-ce que parce qu'un deuxième scénario est de l'ordre du possible, scénario volontariste qui attribue un rôle essentiel à une nouvelle citoyenneté urbaine, par le renforcement du pouvoir et des compétences des villes, le renforcement de l'intercommunalité par rapport au pouvoir cantonal ou confédéral, la recherche de nouveaux liens sociaux et de développement durable axé sur la qualité de vie, l'organisation de l'armature urbaine par le renforcement des pôles intermédiaires centrés sur des réseaux localisés redonnant quelque prééminence à l'espace des lieux sur l'espace des flux.

\section{Réseaux de villes, décentralisation concentrée et développement durable: vers une stratégie de développement territorial à zones de polarisation multiples}

La dimension internationale de l'économie suisse, son orientation vers les marchés européens, son articulation aux réseaux urbains des pays limitrophes exigent une politique d'ouverture au monde mais également une conception d'ensemble de sa politique territoriale. La globalisation des processus de changement, la persistance des disparités internes, la diversité et l'interdépendance des problématiques urbaines et régionales devraient engager la Suisse à recourir à une stratégie de développement territorial d'ensemble et, à renforcer, parallèlement, les outils de régulation existants: la politique régionale, la politique d'environnement et la politique d'aménagement du territoire.

Comme nous l'avons souligné, deux métropoles se constituent en Suisse, regroupant les territoires situés entre Zurich-Olten-Bâle d'une part et entre Genève-Lausanne, Montreux-Yverdon d'autre part. Un aménagement modèle de ces deux régions métropolitaines aurait pour but de faire bénéficier les habitants et les entreprises de tous les avantages de l'urbanité. Ainsi seraient renforcées deux régions qui se maintiendraient au niveau des différentes métropoles voisines, telles que Milan, Munich et Francfort. Si un tel développement est conforme à une certaine logique spatiale et économique à l'œuvre, il est contraire à l'objectif de l'équilibre territorial fédéraliste: on renonce intentionnellement à un développement territorial équilibré.

Une alternative à la métropolisation non maitrisée s`impose. Elle propose de considerer l'ensemble du réseau urbain suisse dans son unité et sa diversité fonctionnelles en développant la complémentarité entre les différents centres urbains de manière à assurer des prestations multiples et de haute qualité à l'ensemble des acteurs sociaux dans un environnement préservé. Un schéma de localisation des services publics privilégiant l'organisation de la Suisse en réseaux de polarisation multiples peut jouer un rôle moteur (ORL, 1993).
Dans le nouveau contexte européen, le mécanisme d'aménagement du territoire mis en place par la Confédération et dont la conception directrice CK 73 était l'élément principal, apparaît plus séduisant que jamais: la décentralisation concentrée de l'urbanisation, la recherche d'une correction des disparités économiques régionales et la procédure de coordination horizontale et verticale étaient à mettre au crédit de cette tentative avortée de planification à l'échelle de l'armature urbaine. Peu optimiste en regard des perspectives d'évolution spontanée, cette conception indiquait quatre nouveaux centres principaux à promouvoir si on voulait éviter que perdurent les tendances lourdes d'une polarisation centralisatrice des activités et des richesses qui prend aujourd'hui la figure polymorphe de la métropolisation. Il s'agissait du couple Bienne-Neuchâtel, du couple Aarau-Olten, de St- Gall (cinq centres qui subissent des processus de désurbanisation relative ou absolue) et de Lucerne (centre principal, Suisse centrale). Quatre autres centres devraient pouvoir assumer en outre au moins une partie des fonctions d'un centre principal et mettre un grand nombre d'équipements et de services performants à la portée des régions périphériques proches: Fribourg, le couple Sion-Sierre, le couple Bellinzone-Lugano, Coire enfin. Cette conception générale pourrait bien être réhabilitée et complétée par des interventions à l'échelle des cantons visant en particulier à aménager des réseaux régionaux et locaux de petites villes et à développer des externalités positives et des effets de connectivité (cf. fig. 6). Dans de nombreuses régions périphériques, le renforcement du potentiel de développement endogène passe sans doute par l'élaboration de politiques volontaristes adaptées à chaque contexte particulier permettant de mettre au point toute une gamme de formules et d'organismes locaux à vocation spécialisée associant le secteur public et le secteur privé. L'aménagement du territoire rencontre ici la nécessité d'une étroite imbrication avec la politique régionale classique de manière à assurer l'amélioration des niveaux de vie et la diversification des activités.

Les régions métropolitaines et urbaines sont des entités économiques et sociales dynamiques qui jouent un rôle moteur dans le développement économique régional, national et international. Mais elles entraînent une dégradation de l'environnement qui se manifeste notamment par la pollution atmosphérique, aquatique, la destruction des sols et la production de grandes quantités de déchets. Ces facteurs, considérés globalement ou séparément, affectent la qualité de vie des habitants. Ces régions urbaines et métropolitaines continueront à jouer un rôle capital dans le changement technologique et dans le développement territorial. Il ne s'agit pas de freiner leur développement mais de maîtriser ses aspects négatifs. La politique d'aménagement du territoire rencontre ici la nécessité d'une intégration avec la politique de l'environnement.

Une des tâches essentielles ouverte à l'action des responsables de la politique d'aménagement et de la politique de l'environnement consiste à faire éclater les décou- 


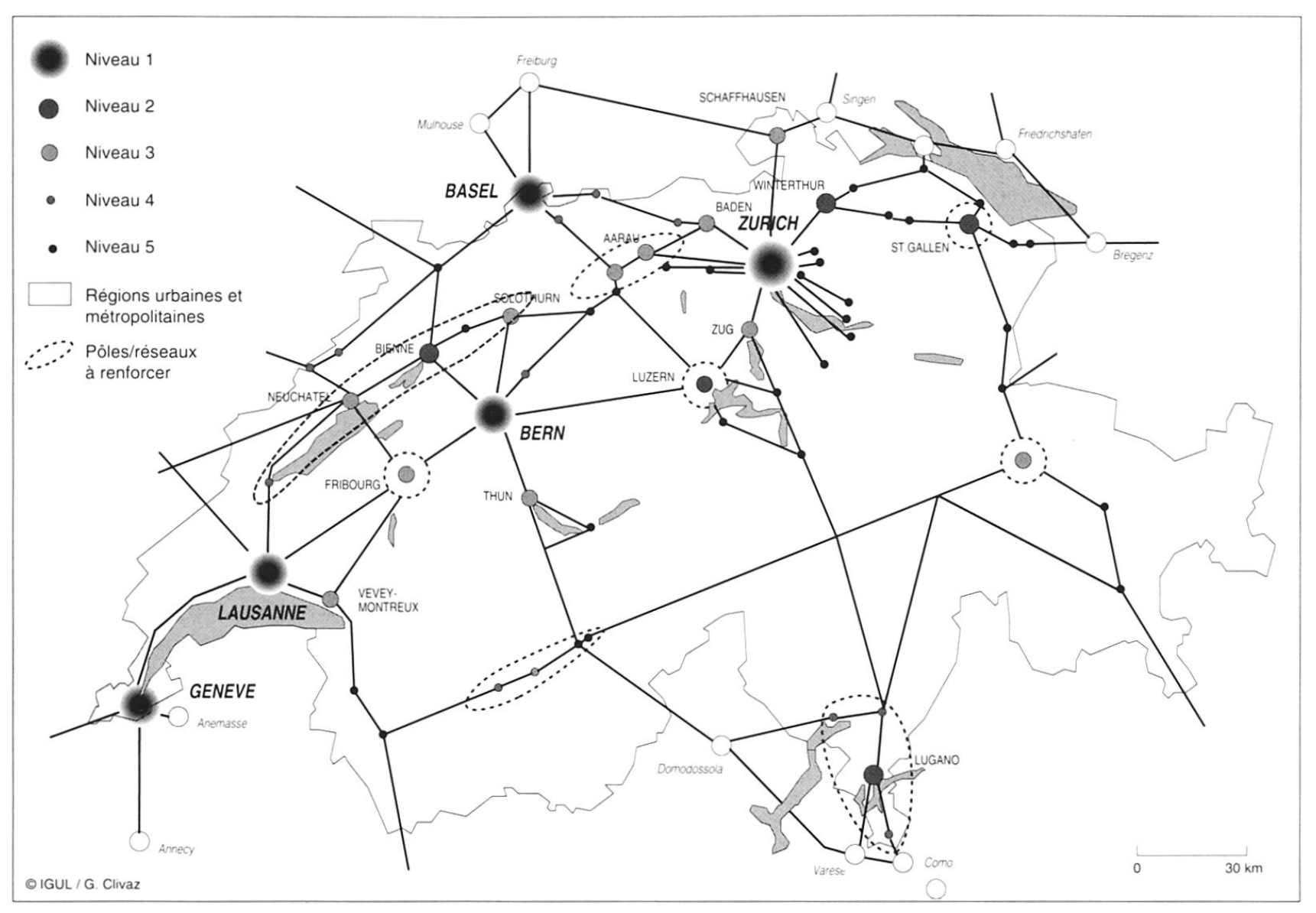

Fig. 6 Hiérarchie urbaine et réseaux de polarité.

pages sectoriels et administratifs qui limitent l'efficacité des interventions visant à améliorer la qualité de vie des citadins et l'équilibre écologique des villes et agglomérations. Le flou ou le vide institutionnel à l'échelle des agglomérations et des régions urbaines constituent de sérieux obstacles à la coopération intercommunale et intercantonale. Le danger de l'absence d'organes de gestion organisés en vue d'ajuster l'échelle des décisions à l'échelle des problèmes est évident. De cette absence il pourrait résulter des formes d'urbanisation relativement fractionnées correspondant aux logiques opératoires mais partielles des acteurs privés et des communes. Les agglomérations et les régions urbaines ou métropolitaines se feraient ainsi à coups de projets partiels et déconnectés, de morceaux de politiques locales sectorielles obéissant à des logiques communales introverties. Une véritable coopération entre les communes qui constituent les zones urbaines doit tôt ou tard se mettre en place si on veut maîtriser les risques et les coûts de la métropolisation et éviter l'absurde d'une urbanisation plus ou moins anarchique. La maîtrise des problèmes environnementaux, la planification des services collectifs, l'aménagement des espaces publics et l'amélioration de l'offre d'accueil sont les quatre enjeux principaux d'une stratégie de croissance qualitative dans les zones à forte nodalité urbaine.

\section{En guise de conclusion: vers une nouvelle régulation territoriale}

Nos observations convergent sur une idée centrale: la nécessité d'une politique visant à une mobilisation du potentiel endogène des régions périphériques et à l'expérimentation de modes de développement urbain alternatifs dans les régions centrales. L'hypothèse d'une bifurcation ou d'un retournement de tendance spontané dans l'évolution des structures régionales dans les années à venir reste improbable. La permanence des disparités et les dangers d'une métropolisation non maîtrisée imposent la continuité et le renforcement d'une politique territoriale, urbaine et régionale, axée sur la croissance qualitative et permettant de corriger progressivement les déséquilibres constatés ou à venir.

Le réseau urbain constitue l'indispensable support de ces politiques. Tous les échelons du réseau hiérarchisé des lieux centraux sont concernés. Cependant, la planification stratégique du territoire devrait avoir comme souci premier la mise en œuvre d'actions différenciées à trois niveaux principaux: les points faibles de l'armature urbaine, les agglomérations et régions urbaines, les réseaux de petits centres périphériques. 
La prise de conscience du caractère multiscalaire et interdépendant des processus de développement urbain et régional réveille la nécessité d'une vision globale et intégrée de la planification territoriale. La nature multiscalaire et multidimensionnelle du développement interdit l'élaboration de recettes passepartout. La planification territoriale est une modalité de régulation globale par la base qui doit encourager la diversité des réponses à des situations locales et régionales également diverses. La régulation contractuelle, décentralisée, stratégique serait désormais le moyen institutionnel permettant de réconcilier la liberté et la mise en cohérence de projets multiples en répondant aux différents défis (économiques, culturels, écologiques, etc.) du développement territorial. Des systèmes de gestion satisfaisants ne peuvent être atteints qu'au travers des circuits décisionnels relativement fermés, décentralisés, qui permettent des autocontrôles rapides et des réactions flexibles aux modifications de l'environnement. Dans un pays fédéraliste, la planification territoriale, ouverte à la sensibilité et aux demandes des différents acteurs sociaux, devrait pouvoir offrir une perspective pertinente à une action des pouvoirs publics axée sur l'ouverture au monde, sur un développement équilibré des villes et des régions fondé sur la valorisation des ressources humaines et sur la protection de l'environnement. C'est bien ce que nous souhaitons à ce pays, et très vite, dans les vingt ans à venir.

\section{Bibliographie}

BASSAND, M., JOYE, D., SCHULER, M. (1988): L'urbanisation de la Suisse: faits et perspectives. Dans: Les enjeux de l'urbanisation, OEPR, Peter Lang, Bern, p. 51-69.
BERRY, B. J. L. (1973): The Human Consequences of Urbanisation. London and Basingstoke, Macmillan.

CUNHA, A. (1993): Développement territorial, régions et centralité urbaine: le cas Suisse. Thèse présentée à l'Ecole des Hautes Etudes Commerciales de l'Université de Lausanne.

CUNHA, A., RACINE, J.-B. (1994): Changement structurel et évolution du réseau urbain helvétique, Rapport de recherche, FNRS (à paraître).

DANIELS, P. (1993): Activités tertiaires et dynamique des métropoles: comparaisons internationales. Dans: Métropoles en déséquilibre?, Economica, p. 397-430

GAUDARD, G. (1974): Le problème des limites de la croissance et l'espace économique. Dans: Informations, BPS no 59, p. 3-15.

KLAASSEN, L., SCIMENI, G. (1981): Theoretical issues in urban dynamics. Dans: KLAASSEN, L., MOLLE, W., PAELINCK, J.: The Urban Development, Aldershot, Gower, p. 12-28.

ORL (1993): Grundzüge der Raumordnung Schweiz, Zürich, ETH.

RACINE, J.-B. (1992): Développement urbain métropolitain et crise de la ville: un monde en transition. Dans: Revue suisse d'économie politique et de Statistique, vol. 128(3), 241-260.

ROSSI, A. (1983): La décentralisation urbaine en Suisse. Lausanne, Presses polytechniques romandes.

ROSSI, A., et PINI, G. (1992): Entreprises et système urbain. Rapport de recherche, FNRS, PNR 25 (à paraître).

SCHULER, M. (1994): Les Différents Niveaux Géographiques de la Suisse. Berne, OFS

TOURAINE, A. (1991): Face à l'exclusion. Dans: BAUDRILLARD, J., LIPOVETSKY, G., et al. Citoyenneté et urbanité, Paris, Esprit.

WACKERMANN, G. (1993): Nouveaux moteurs de la mobilité des firmes et internationalisation des échelles spatiales. Dans: Annales de la Géographie, no 557, p. 31-52. 